\title{
RANTES/CCL5 gene polymorphisms, serum concentrations, and incident type 2 diabetes: results from the MONICA/KORA Augsburg case-cohort study, 1984-2002
}

\author{
Christian Herder, Thomas Illig ${ }^{1}$, Jens Baumert ${ }^{1}$, Martina Müller ${ }^{1,2}$, Norman Klopp ${ }^{1}$, Natalie Khuseyinova ${ }^{3}$, \\ Christa Meisinger ${ }^{1}$, Ulrike Poschen, Stephan Martin, Wolfgang Koenig ${ }^{3}$ and Barbara Thorand ${ }^{1}$ \\ Institute for Clinical Diabetes Research, German Diabetes Centre, Leibniz Institute at Heinrich Heine University, 40225 Düsseldorf, Germany, ${ }^{1}$ Institute of \\ Epidemiology, Helmholtz Zentrum München, German Research Centre for Environmental Health, 85764 Neuherberg, Germany, ${ }^{2}$ Institute of Medical \\ Information Processing, Biometry and Epidemiology, Chair of Epidemiology, Ludwig-Maximillians-Universität, 81377 München, Germany and \\ ${ }^{3}$ Department of Internal Medicine II-Cardiology, University of Ulm Medical Center, Robert-Koch-Street 8, 89081 Ulm, Germany \\ (Correspondence should be addressed to W Koenig; Email: wolfgang.koenig@uniklinik-ulm.de)
}

\begin{abstract}
Objective: Regulated on activation, normal T-cell expressed and secreted (RANTES)/chemokine(C-C motif) ligand (CCL5) is expressed by adipocytes, and serum levels of RANTES are increased in obesity and type 2 diabetes. The aim of this study was to test the hypothesis that RANTES is involved in the pathogenesis of type 2 diabetes by analyzing the triangular association between CCL5 gene polymorphisms, systemic RANTES concentrations, and incident type 2 diabetes in a large prospective study.

Subjects and methods: The study is based on 502 individuals (293 men and 209 women) and 1632 individuals (859 men and 773 women) with and without incident type 2 diabetes from the populationbased MONItoring of Trends and Determinants in Cardiovascular Disease (MONICA)/Cooperative Health Research in the Region of Augsburg (KORA) case-cohort study respectively (mean follow-up time \pm s.D. $10.1 \pm 4.9$ years). CCL5 genotypes and RANTES serum concentrations were determined and associations between genotypes, haplotypes, serum levels, and incident type 2 diabetes were assessed.

Results: Minor alleles of four single nucleotide polymorphisms were associated with lower RANTES levels $\left(P_{\text {additive }}\right.$ between $1.2 \times 10^{-9}$ and $3.1 \times 10^{-8}$ ), but neither genotypes, haplotypes, nor serum levels were associated with incident type 2 diabetes.

Conclusions: Our data suggest that RANTES/CCL5 gene variants and serum levels are not causally related with type 2 diabetes and that elevated RANTES levels in patients with type 2 diabetes may be a consequence of hyperglycemia. However, our findings cannot preclude a local role in adipose tissue where RANTES expression may contribute to leukocyte infiltration and a proinflammatory state.
\end{abstract}

European Journal of Endocrinology 158 R1-R5

\section{Introduction}

Regulated on activation, normal T-cell expressed and secreted (RANTES)/CCL5 belongs to the family of CC chemokines that recruit and activate different subtypes of leukocytes such as T cells, monocytes, basophils, eosinophils, or mast cells (1). RANTES is expressed by adipocytes and has been hypothesized to mediate leukocyte infiltration of adipose tissue in obesity $(2,3)$. Circulating RANTES concentrations are elevated in obesity, impaired glucose tolerance (IGT), and type 2 diabetes $(4,5)$. In the Finnish Diabetes Prevention Study, high RANTES levels were associated with resistance to lifestyle intervention and higher incidence of type 2 diabetes in the intervention group (6). Thus, the aim of this study was to analyze whether RANTES may be involved in the pathogenesis of type 2 diabetes by investigating the triangular relationship between
CCL5 gene polymorphisms, RANTES serum levels, and incident type 2 diabetes in the population-based MONItoring of Trends and Determinants in Cardiovascular Disease (MONICA)/Cooperative Health Research in the Region of Augsburg (KORA) case-cohort study. This study is based on a Mendelian randomization approach. Any association between circulating RANTES concentrations and incident type 2 diabetes could be attributable to reverse causation or residual confounding factors. However, the additional analysis of the link between genotypes, serum levels, and type 2 diabetes incidence could reveal whether RANTES really plays a causal role in the development of type 2 diabetes because genotypes that are associated with high RANTES levels are randomly transmitted to carriers from their parents and potentially confounding factors should be distributed evenly across genotypes. 


\section{Subjects and methods}

\section{Subjects}

The present study is based on the population-based MONICA/KORA case-cohort study. This prospective study (7-9) includes 2134 participants (293 men and 209 women with incident type 2 diabetes; 859 men and 773 women without incident type 2 diabetes). Participants with self-reported diabetes at baseline were excluded. The incidence of type 2 diabetes was assessed using a written follow-up questionnaire sent to all participants of the three baseline surveys in 1997/1998 and 2002/2003. Furthermore, all subjects who participated in S1 were invited to a follow-up examination in $1987 / 1988$. Cases with self-reported incident diabetes were validated by a questionnaire mailed to the treating physician or medical chart review. Only subjects for whom the treating physician clearly reported a diagnosis of type 2 diabetes or for whom a diagnosis of type 2 diabetes was mentioned in the medical records, or who were taking antidiabetic medication were classified as cases for the present analysis. Time of incidence was generally based on the information from the treating physician if the physician reported that he/she had diagnosed the disease himself/herself. If no information could be obtained from the physician who had first diagnosed the disease, the date of diagnosis as reported by the study participant was used. Mean follow-up time $( \pm$ s.D.) was $10.1 \pm 4.9$ years. For analyses with incident type 2 diabetes as the endpoint, DNA was available from 2067 participants (1142 men and 925 women). For cross-sectional analyses in a random sample of the total study population, 1872 participants (1001 men and 871 women) with DNA samples and complete information on covariables were available. The study was approved by the local authorities, and all participants provided written informed consent.

\section{Measurement of RANTES serum concentrations}

RANTES serum concentrations were measured with the Quantikine ELISA kit (R\&D Systems, Wiesbaden, Germany). Mean intra- and inter-assay coefficient of variation $(\mathrm{CV})$ values were 4.2 and $5.1 \%$ respectively, for recombinant controls, and 4.7 and $6.4 \%$ respectively, for test sera.

\section{Genotyping}

In order to obtain complete coverage of the CCL5 gene, six single nucleotide polymorphisms (SNPs) were selected on the basis of data from the International HapMap project (http://www.hapmap.org) in May 2005 for a $9.2 \mathrm{~kb}$ locus of the CCL5 gene (promoter: rs2107538 (-403G>A), rs2280788 (-28C >G); intronic: rs2280789, rs4796120, rs3817655; 3 '-flanking region: rs1065341) and covered the total common variation in the gene at the time of SNP selection. Genotyping was carried out with matrixassisted laser desorption-ionization time of flight analysis of allele-dependent primer extension products, as reported previously (9). A subset of samples (13.3\%) was genotyped in duplicate to assess genotyping quality. The mean discordance rate for the six SNPs was $0.44 \%$.

\section{Statistical analysis}

Identification of haplotype blocks and statistical analysis were performed as described (9). The associations between RANTES levels or genotypes and incident type 2 diabetes were assessed using Cox proportional hazards analysis. As RANTES concentrations were lognormal distributed, the geometric mean adjusted for age, sex, and survey was computer stratified by CCL5 genotypes. Pairwise linkage disequilibrium was measured using Lewontin's $D^{\prime}$ and the squared correlation coefficient $r^{2}$ (Fig. 1 of the online appendix which can be viewed online at http://www.eje-online. org/supplemental/).

In general, $P<0.05$ was considered statistically significant. In our analysis of the associations between the six CCL5 SNPs and serum levels or incident type 2 diabetes, we calculated the number of effectively independent tests from the correlation structure between the SNPs (10). Given five effective SNPs, a priori planned sex-stratified analyses and two outcomes (serum levels and type 2 diabetes), the significance level adjusted for multiple testing according to Bonferroni for each of the SNPs was 0.0025 in each analysis. All evaluations were performed with the statistical software package SAS (Version 8.02 for Unix, Version 9.1 for Windows, SAS Institute, Cary, NC, USA) and the statistical analysis software R, Version 2.4.1 (11).

\section{Results}

The baseline characteristics of the MONICA/KORA case-cohort study population have been reported before (9) (Table 1 of the online appendix which can be viewed online at http://www.eje-online.org/supplemental/). CCL5 genotype frequencies for the randomly sampled subcohort are shown in Table 1. Genotyping success rates were 97.7, 98.3, 97.8, 98.2, 98.1, and $97.3 \%$ for rs2107538, rs2280788, rs2280789, rs4796120, rs3817655, and rs1065341 respectively. All SNPs were in Hardy-Weinberg equilibrium $(P>0.2)$ and lie in the same haplotype block $\left(D^{\prime}>0.98\right.$ between consecutive SNPs; Fig. 1 of the online appendix which can be viewed online at http://www.eje-online.org/ supplemental/). Rs3817655 and rs2107538 were highly correlated $\left(r^{2}=0.977\right)$ and provided rather similar information. All following analyses were a priori 
Table 1 Frequencies of CCL5 genotypes in the randomly sampled subcohort $(n=1872)$ and hazard ratios $(95 \% \mathrm{Cl})$ for incident type 2 diabetes comparing CCL5 genotypes from Cox proportional hazards models $(n=2067)$.

\begin{tabular}{|c|c|c|c|c|c|c|c|}
\hline \multirow[b]{2}{*}{ SNP } & \multirow{2}{*}{$\begin{array}{c}\text { Allele }^{a} \\
1 / 2\end{array}$} & \multirow{2}{*}{$\begin{array}{c}n^{\mathrm{a}} \\
11 / 12 / 22\end{array}$} & \multirow{2}{*}{$\begin{array}{c}\text { Weighted frequency }(\%)^{\mathrm{a}} \\
11 / 12 / 22\end{array}$} & \multirow[b]{2}{*}{ Model $^{b}$} & \multicolumn{3}{|c|}{ Genotype $^{a}$} \\
\hline & & & & & 11 & 12 & 22 \\
\hline \multirow[t]{3}{*}{ rs2107538 } & $\mathrm{C} / \mathrm{T}$ & $1212 / 560 / 64$ & $65.8 / 30.6 / 3.6$ & 1 & 1 (ref) & $0.88(0.70-1.10)$ & $0.91(0.51-1.64)$ \\
\hline & & & & 2 & 1 (ref) & $0.83(0.65-1.07)$ & $0.88(0.46-1.67)$ \\
\hline & & & & 3 & 1 (ref) & $0.84(0.65-1.08)$ & $0.95(0.49-1.85)$ \\
\hline \multirow[t]{3}{*}{ rs2280788 } & $\mathrm{C} / \mathrm{G}$ & $1761 / 78 / 0$ & $95.8 / 4.2 / 0$ & 1 & 1 (ref) & $0.67(0.37-1.21)$ & $N / A$ \\
\hline & & & & 2 & 1 (ref) & $0.77(0.42-1.44)$ & N/A \\
\hline & & & & 3 & 1 (ref) & $0.88(0.48-1.61)$ & $N / A$ \\
\hline \multirow{3}{*}{ rs2280789 } & $\mathrm{T} / \mathrm{C}$ & $1397 / 412 / 35$ & $75.7 / 22.5 / 1.9$ & 1 & 1 (ref) & $0.94(0.74-1.21)$ & $0.46(0.15-1.35)$ \\
\hline & & & & 2 & 1 (ref) & $0.97(0.74-1.26)$ & $0.44(0.14-1.38)$ \\
\hline & & & & 3 & 1 (ref) & $1.01(0.77-1.32)$ & $0.45(0.14-1.51)$ \\
\hline \multirow[t]{3}{*}{ rs4796120 } & $A / G$ & $1469 / 353 / 25$ & $79.5 / 19.2 / 1.4$ & 1 & 1 (ref) & $1.04(0.81-1.35)$ & $0.40(0.12-1.38)$ \\
\hline & & & & 2 & 1 (ref) & $1.05(0.80-1.38)$ & $0.36(0.10-1.32)$ \\
\hline & & & & 3 & 1 (ref) & $1.07(0.81-1.41)$ & $0.38(0.10-1.48)$ \\
\hline \multirow[t]{3}{*}{ rs3817655 } & T/A & $1209 / 564 / 68$ & $65.5 / 30.7 / 3.7$ & 1 & 1 (ref) & $0.89(0.71-1.11)$ & $0.89(0.50-1.58)$ \\
\hline & & & & 2 & 1 (ref) & $0.85(0.66-1.09)$ & $0.86(0.46-1.61)$ \\
\hline & & & & 3 & 1 (ref) & $0.86(0.67-1.10)$ & $0.94(0.49-1.80)$ \\
\hline \multirow[t]{3}{*}{ rs1065341 } & $A / G$ & $1,634 / 196 / 9$ & $88.8 / 10.7 / 0.5$ & 1 & 1 (ref) & $1.01(0.72-1.42)$ & $0.52(0.13-2.05)$ \\
\hline & & & & 2 & 1 (ref) & $0.87(0.58-1.29)$ & $0.53(0.15-1.89)$ \\
\hline & & & & 3 & 1 (ref) & $0.85(0.57-1.26)$ & $0.54(0.14-2.09)$ \\
\hline
\end{tabular}

N/A, not applicable (no homozygous carriers of the minor allele); ref, reference genotype.

${ }^{a}$ Allele 1 denotes the major allele and allele 2 denotes the minor allele.

${ }^{\mathrm{b}}$ Model 1, adjusted for age, sex, and survey; Model 2, Model 1+ BMI; Model 3, Model 2+ smoking status, alcohol intake, physical activity, systolic blood pressure, and ratio of total cholesterol:HDL cholesterol.

planned and performed separately for men and women, but as no sex differences were detected, combined results are given.

First, we investigated whether CCL5 genotypes have an impact on RANTES levels and found that the minor alleles of four SNPs were associated with lower serum levels $\left(P_{\text {additive }}\right.$ between $1.2 \times 10^{-9}$ and $3.1 \times 10^{-8}$; Fig. 1A). The CCL5 haplotype TCCGAA that contained these four minor alleles was also associated with significantly lower serum levels of RANTES compared with most frequent haplotype CCTATA $\left(P=4.9 \times 10^{-9}\right.$; see also Table 3 of the online appendix which can be viewed online at http://www.eje-online.org/supplemental/). In the age-, sex-, and survey-adjusted analyses, RANTES levels for one or two copies of the TCCGAA haplotype were 24445 and $20561 \mathrm{pg} / \mathrm{ml}$ respectively, compared with $29063 \mathrm{pg} / \mathrm{ml}$ for two copies of the CCTATA haplotype (calculated for a 60 -year-old male participant in survey 1 ).

Second, we analyzed the association between RANTES levels and the risk for type 2 diabetes. In the unadjusted analysis, RANTES levels at baseline were slightly higher in cases than in non-cases (weighted geometric means 25420 and $23807 \mathrm{pg} / \mathrm{ml}$ respectively; $P=0.029$, see also Table 1 of the online appendix which can be viewed online at http://www.eje-online.org/supplemental/). Figure 1B shows hazard ratios (HRs) for incident type 2 diabetes across quartiles of RANTES levels from Cox proportional hazards models with different degrees of adjustment for the potential confounders. When compared with the lowest RANTES quartile, higher RANTES concentrations were not significantly associated with increased risk to develop type 2 diabetes during follow-up. In addition, we assessed the association between continuous RANTES levels and the incidence of type 2 diabetes. A 1-SD increase of RANTES was associated with incident type 2 diabetes only in the age-, sex-, and survey-adjusted model (HR (95\% confidence interval (CI) 1.12 (1.02-1.24); $P=0.024)$, but was lost when including body mass index (BMI) (HR (95\% CI) 1.03 (0.91-1.16); $P=0.689)$ into the model. Furthermore, adjustment for other potential confounders had almost no impact on the BMI-adjusted HR (data not shown).

Third, all SNPs and haplotypes calculated from them were tested for association with incident type 2 diabetes. None of the SNPs or haplotypes were significantly associated with diabetes risk (Table 1; Tables 2 and 3 of the online appendix which can be viewed online at http://www.eje-online.org/supplemental/).

\section{Discussion}

This is the first population-based study to investigate the triangular relationship between CCL5 gene variants, circulating RANTES levels, and incident type 2 diabetes. Several CCL5 alleles were strongly related to decreased RANTES levels, but neither CCL5 genotypes nor RANTES concentrations were associated with incident type 2 diabetes. Our data corroborate and extend findings from smaller studies that suggested an association between CCL5 genotypes and circulating RANTES levels $(12,13)$. In addition, our data are in line with previous findings in the Finnish Diabetes Prevention Study. High RANTES levels were not associated with elevated risk for type 2 diabetes in the control 

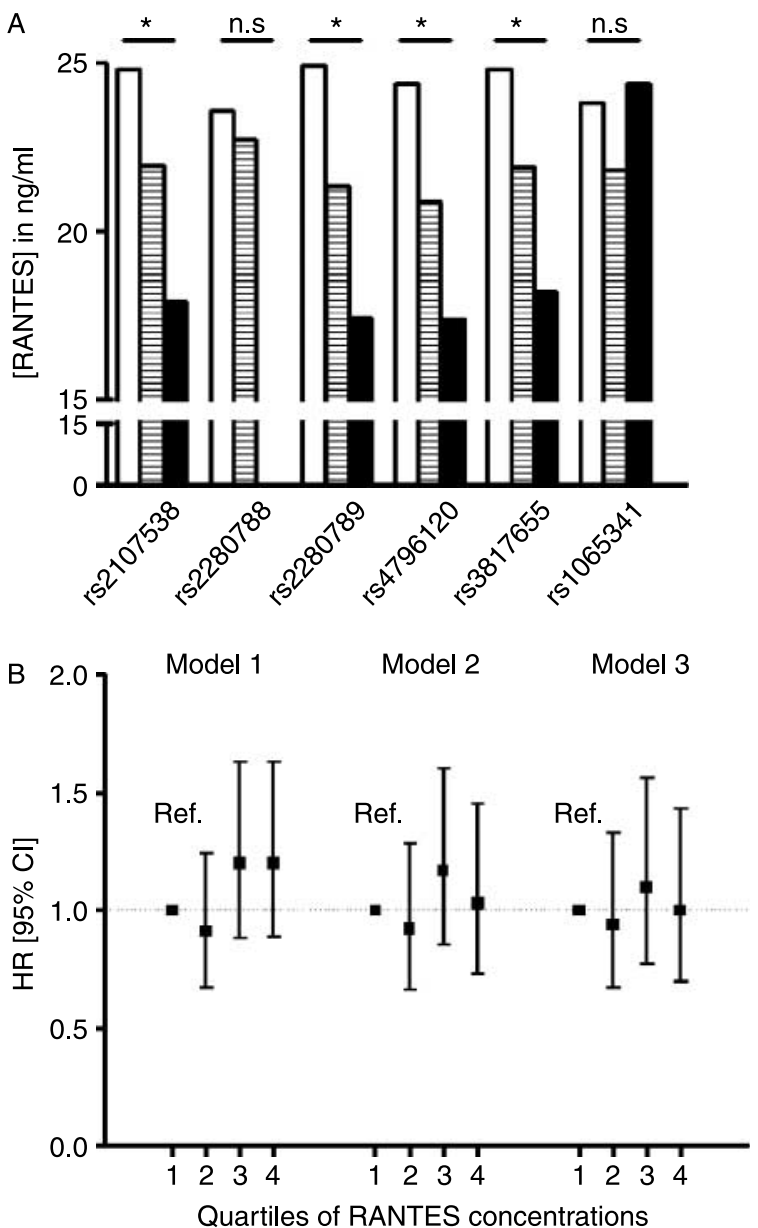

Figure 1 (A) Serum concentrations of RANTES (geometric means) stratified by CCL5 genotype in the randomly drawn subcohort $(n=1872)$. Open bars, hatched bars, and black bars indicate homozygous carriers of the major allele, heterozygotes, and homozygous carriers of the minor allele respectively. NS, not significant; * $P_{\text {additive }} \leq 3.1 \times 10^{-8}$. (B) Hazard ratios $(95 \% \mathrm{Cl})$ of developing type 2 diabetes comparing quartiles of RANTES serum concentrations. Model 1, adjusted for age, sex, and survey; Model 2 , adjusted for age, sex, survey, and BMI; Model 3, adjusted for age, sex, survey, BMI, systolic blood pressure, ratio of total cholesterol: HDL cholesterol, physical activity (active/inactive), alcohol intake $(0 />0$ to $39.9 / \geq 40 \mathrm{~g} / \mathrm{d}$ for men and $0 />0$ to $19.9 / \geq 20 \mathrm{~g} / \mathrm{d}$ for women), smoking status (current, former, never smoker), and parental type 2 diabetes (positive, unknown, negative). If not indicated otherwise, variables were included in continuous form. Ref., reference quartile.

group which may be comparable with our group of study participants (6). However, high RANTES levels were predictive of incident type 2 diabetes in the intervention group which indicates that for as yet unknown reasons individuals with lower RANTES levels could be more responsive to intensive lifestyle intervention than individuals with higher RANTES levels (6).

Strengths of the study include the use of the MONICA/KORA cohort with a large number of cases and non-cases, long follow-up, and detailed information on immune markers and disease risk. Our study had a power to detect an association with a HR of 1.5 of RANTES serum levels with incident type 2 diabetes of $98 \%$ assuming an exposition frequency of $25 \%$ as calculated a priori. We chose a HR of 1.5 for the power calculation because we found previously in the same study population that HRs for the comparison of extreme quartiles or tertiles of other immune mediators such as C-reactive protein, interleukin-6, interleukin-18, monocyte chemoattractant protein- 1 , and macrophage migration inhibitory factor were often $\approx 1.5$ or higher $(7-9,16)$.

However, statistical power was lower for the genetic analyses. Assuming HRs of 1.3, our study had a power of 78,68 , and $47 \%$ to detect an association between CCL5 genotypes with diabetes risk in a dominant model with a frequency of 30,20 , and $10 \%$ respectively of the genotype with at least one minor allele (post hoc power calculation). Thus, we cannot exclude that we missed weaker associations between CCL5 genotypes and incident type 2 diabetes in this study.

Taken together, our findings indicate that elevated RANTES levels in IGT and type 2 diabetes as reported before $(4,5)$ may represent a consequence rather than cause of hyperglycemia. However, our data do not preclude a local pathogenetic role in adipose tissue where RANTES expression may contribute to leukocyte chemotaxis and infiltration. Furthermore, it remains to be seen whether RANTES may be more relevant for the risk of diabetic complications than for type 2 diabetes itself, as previous studies demonstrated associations of CCL5 variants with diabetic neuropathy (14) and cardiovascular disease $(13,15)$.

\section{Acknowledgements}

The MONICA/KORA Augsburg cohort study was financed by the GSF-National Research Center for Environment and Health now Helmholtz Zentrum München, German Research Center for Environmental Health and supported by grants from the Federal Ministry of Education and Research. The present study was funded by the German Federal Ministry of Education, Science, Research and Technology (National Genome Research Net-2, cardiovascular, 01GS0423), the German Federal Ministry of Health and Social Security, the Ministry of Science and Research of the State of North Rhine-Westphalia and the German Research Foundation (TH 784/2-1). We thank all members of the GSF-Institute of Epidemiology who were involved in the planning and conduct of the MONICA/KORA Augsburg studies and the MONICA Augsburg survey team. We are grateful to Prof. L Chambless (School of Public Health, University of North Carolina at Chapel Hill, NC, USA) for statistical advice concerning the analysis of the case-cohort dataset and to Melanie Kolz (GSF) for advice regarding the genotyping. Finally, we are indebted to all study participants. 


\section{References}

1 Appay V \& Rowland-Jones SL. RANTES: a versatile and controversial chemokine. Trends in Immunology 200122 83-87.

2 Poulain-Godefroy $\mathrm{O} \&$ Froguel P. Preadipocyte response and impairment of differentiation in an inflammatory environment. Biochemical and Biophysical Research Communications 2007356 662-667.

$3 \mathrm{Wu} \mathrm{H}$, Ghosh S, Perrard XD, Feng L, Garcia GE, Perrard JL, Sweeney JF, Peterson LE, Chan L, Smith CW \& Ballantyne CM. T-cell accumulation and regulated on activation, normal $\mathrm{T}$ cell expressed and secreted upregulation in adipose tissue in obesity. Circulation 2007115 1029-1038.

4 Nomura S, Shouzu A, Omoto S, Nishikawa M \& Fukuhara S. Significance of chemokines and activated platelets in patients with diabetes. Clinical and Experimental Immunology $2000 \mathbf{1 2 1}$ 437-443.

5 Herder C, Haastert B, Muller-Scholze S, Koenig W, Thorand B, Holle R, Wichmann HE, Scherbaum WA, Martin S \& Kolb H. Association of systemic chemokine concentrations with impaired glucose tolerance and type 2 diabetes: results from the Cooperative Health Research in the Region of Augsburg, Survey S4 (KORA S4). Diabetes 200554 (Supplement 2) S11-S17.

6 Herder C, Peltonen M, Koenig W, Kraft I, Muller-Scholze S, Martin S, Lakka T, Ilanne-Parikka P, Eriksson HG, Hamalainen H, Keinanen-Kiukaanniemi S, Valle TT, Uusitupa M, Lindstrom J, Kolb H \& Tuomilehto J. Systemic immune mediators and lifestyle changes in the prevention of type 2 diabetes: results from the Finnish Diabetes Prevention Study. Diabetes 200655 2340-2346.

7 Herder C, Baumert J, Thorand B, Koenig W, de Jager W, Meisinger C, Illig T, Martin S \& Kolb H. Chemokines as risk factors for type 2 diabetes: results from the MONICA/KORA Augsburg Study, 1984-2002. Diabetologia 200649 921-929.

8 Thorand B, Baumert J, Kolb H, Meisinger C, Chabless L, Koenig W \& Herder C. Sex differences in the prediction of type 2 diabetes by inflammatory markers: results from the MONICA/KORA Augsburg case-cohort study, 1984-2002. Diabetes Care 2007 30 854-860.
9 Herder C, Klopp N, Baumert J, Muller M, Khuseyinova N, Meisinger C, Martin S, Illig T, Koenig W \& Thorand B. Effect of macrophage migration inhibitory factor (MIF) gene variants and MIF serum concentrations on the risk of type 2 diabetes: results from the MONICA/KORA Augsburg case-cohort study, 19842002. Diabetologia $2008 \mathbf{5 1} 276-284$.

$10 \mathrm{Li} \mathrm{J} \mathrm{\&} \mathrm{Ji} \mathrm{L.} \mathrm{Adjusting} \mathrm{multiple} \mathrm{testing} \mathrm{in} \mathrm{multilocus} \mathrm{analyses} \mathrm{using}$ the eigenvalues of a correlation matrix. Heredity 200595 221-227.

11 R Development Core Team. R: a language and environment for statistical computing. R Foundation for Statistical Computing, Vienna, Austria. ISBN 3-900051-07-0, URL http://www. R-project.org, 2006.

12 Zhernakova A, Alizadeh BZ, Eerligh P, Hanifi-Moghaddam P, Schloot NC, Diosdado B, Wijmenga C, Roep BO \& Koeleman BPC. Genetic variants of RANTES are associated with serum RANTES levels and protection for type 1 diabetes. Genes and Immunity 2006 7 544-549.

13 Jang Y, Chae JS, Hyun YJ, Koh SJ, Kim JY, Ko MJ, Rim SJ, Shin HJ, Ordovas JM \& Lee JH. The RANTES $-403 \mathrm{G}>\mathrm{A}$ promoter polymorphism in Korean men: association with serum RANTES and coronary artery disease. Clinical Science $2007113349-356$.

14 Nakajima K, Tanaka Y, Nomiyama T, Ogihara T, Ikeda F, Kanno R, Iwashita N, Sakai K, Watada H, Onuma T \& Kawamori R. RANTES promoter genotype is associated with diabetic nephropathy in type 2 diabetic subjects. Diabetes Care 200326 892-898.

15 Simeoni E, Winkelmann BR, Hoffmann MM, Fleury S, Ruiz J, Kappenberger L, März W \& Vassalli G. Association of RANTES G-403A gene polymorphism with increased risk of coronary arteriosclerosis. European Heart Journal 200425 1378-1381.

16 Thorand B, Kolb H, Baumert J, Koenig W, Chambless W, Meisinger C, Illig T, Martin S \& Herder C. Elevated levels of interleukin- 18 predict the development of type 2 diabetes: results from the MONICA/KORA Augsburg Study, 1984-2002. Diabetes $2005542932-2938$.

Received 23 January 2008

Accepted 31 January 2008 